\title{
Charge amplification concepts for direction-sensitive dark matter detectors
}

\author{
D. Dujmic ${ }^{c, d, *}$ P. Fisher ${ }^{c, d, e}$ G. Sciolla ${ }^{c}$ S. Ahlen ${ }^{a}$ V. Dutta ${ }^{c}$ \\ S. Henderson ${ }^{c}$ A. Kaboth ${ }^{c}$ G. Kohse ${ }^{f}$ R. Lanza ${ }^{\text {g }}$ J. Monroe ${ }^{c}$ \\ A. Roccaro ${ }^{a}$ N. Skvorodnev ${ }^{b}$ H. Tomita ${ }^{a}$ R. Vanderspek ${ }^{c}$ \\ H. Wellenstein ${ }^{b}$ R. Yamamoto ${ }^{c}$ \\ a Physics Department, Boston University, Boston, MA 02215 \\ ${ }^{\mathrm{b}}$ Physics Department, Brandeis University, Waltham, MA 02454 \\ ${ }^{\mathrm{c}}$ Department of Physics, MIT, Cambridge, MA 02139 \\ ${ }^{\mathrm{d}}$ Laboratory for Nuclear Science, MIT, Cambridge, MA 02139 \\ e MIT Kavli Institute for Astrophysics and Space Research, Cambridge, MA 02139 \\ ${ }^{\mathrm{f}}$ Nuclear Reactor Laboratory, MIT, Cambridge, MA 02139 \\ ${ }^{g}$ Department of Nuclear Science and Engineering, MIT, Cambridge, MA 02139
}

\begin{abstract}
Direction measurement of weakly interacting massive particles in time-projection chambers can provide definite evidence of their existence and help to determine their properties. This article demonstrates several concepts for charge amplification in time-projection chambers that can be used in direction-sensitive dark matter search experiments. We demonstrate reconstruction of the "head-tail" effect for nuclear recoils above $100 \mathrm{keV}$, and discuss the detector performance in the context of dark matter detection and scaling to large detector volumes.
\end{abstract}

Key words: Dark Matter, Directional Detector, Nuclear Scattering, Optical Readout, TPC, WIMP PACS: 29.40.Cs, 29.40.Gx, 95.35.+d

\footnotetext{
* Corresponding author.

Email address: ddujmic@mit.edu (D. Dujmic).
} 


\section{Introduction}

Weakly interacting massive particles (WIMPs) are good candidates for constituents of a dark-matter halo around our galaxy, and a target of several experimental searches (see e.g. [1] for review). An annual oscillation in the signal at the level of 1-2\% was recently confirmed by DAMA collaboration [2]. If the asymmetry is due to Earth's motion around Sun then much larger diurnal asymmetry in the WIMP direction is expected [5]. However, the DAMA and other experiments that report zero signal [3, 4] use liquid and solid detector materials that allow for more compact detectors, but destroy information on the WIMP direction and the sense of direction ("head-tail"). Therefore, directionality is particularly valuable, both for suppressing background and for confirming the DAMA result by correlating a candidate dark-matter signal with astrophysical phenomena.

Reconstruction of the WIMP direction can be accomplished with detectors using low-pressure gas as the target material. Several groups, DRIFT [6], NEWAGE [7], MIMAC [8] and DMTPC [9] have reconstructed low-momentum recoils created in elastic neutron scattering using low-pressure time-projection chambers for dark matter searches. The reconstruction of the sense of direction ("head-tail") using the scintillation profile of a recoiling track has been only recently achieved [9].

The low density of gaseous detectors and the small WIMP cross section necessitate the use of large detector volumes with fine detector granularities. A significant improvement of the current experimental limits may require a ton-scale detector. In the case of $\mathrm{CF}_{4}$ as the detector material, one ton of gas occupies a volume of approximately $16 \times 16 \times 16 \mathrm{~m}^{3}$ at 50 Torr of pressure. A $50 \mathrm{keV}$ fluorine recoil created in a WIMP collision travels $1.5 \mathrm{~mm}$ at 50 Torr of pressure. Therefore, a multi-cubic meter detector with resolution of the order of hundreds of micrometers is needed for a directional dark matter experiment, requiring further progress in detector technology in order to observe WIMPs.

\section{Detector designs}

In this paper we propose and demonstrate several designs for time-projection chambers that can be used in large-volume, directional dark matter search experiments with "head-tail" discrimination. In a time projection chamber, a WIMP creates a nuclear recoil that makes electron-ion pairs as it slows down in the detector gas. We use $\mathrm{CF}_{4}$ gas that has good charge multiplication and scintillation properties [10,11,12. Fluorine, in addition, has nonzero angular momentum, which allows probing for spin-dependent dark mat- 
ter interactions. The electron diffusion limits the maximum drift distance to about $25 \mathrm{~cm} \mathrm{[7,9].} \mathrm{The} \mathrm{initial} \mathrm{ionization} \mathrm{electrons} \mathrm{drift} \mathrm{in} \mathrm{electric} \mathrm{field} \mathrm{to-}$ ward the charge-amplification region, where in case of $\mathrm{CF}_{4}$, the threshold for charge multiplication is approximately $45 \mathrm{~V} /(\mathrm{cm} \cdot$ Torr) [10]. Traditionally, design choices for amplification regions have been based on the multiwire proportional chamber (MWPC) [13], where strong electric fields are created in the vicinity of thin wires. The pitch between wires, and therefore the spatial resolution in MWPC is limited to above 1-2 mm due to mechanical and electrostatic reasons. Finer spatial resolution can be achieved with micropattern detectors (e.g. [14]), but the size and the gain of detector modules are constrained by manufacturing limitations.

In this paper we demonstrate designs for micropattern detectors that provide good gain, fine granularity and scalability to large volumes. Electrodes used in the charge multiplication are made of woven meshes, indium-tin-oxide (ITO) films, or copper. All designs allow production of large-area modules as the meshes and ITO foils are produced in $1.2 \mathrm{~m}$-wide rolls. In the first case shown in Figure 1a), the amplification region is made of a stainless-steel mesh and copper-clad G10 board. The stainless steel mesh is made of $28 \mu \mathrm{m}$ diameter wires with periodicity of $256 \mu \mathrm{m}$, which gives optical transmittance of $77 \%$. Woven meshes are used in air filtration systems and can be mass produced in a cost-effective way. The mesh and the copper-clad board are separated by fluorocarbon resistive (fishing) wires of $0.54 \mathrm{~mm}$ diameter and spaced every $2 \mathrm{~cm}$, with transparency of $97 \%$. With this design, we demonstrate the reconstruction of $2 \mathrm{D}$ recoil segments with improved gain and no additional cost compared to our previous detector. Further improvement in gain may be achievable with the use of transparent electrodes that allow scintillation light to be read out from two sides. We test a design shown in Figure 1 $1 \mathrm{~b}$ ), where the amplification electrodes are made of ITO layers deposited on mylar foil and a stainless steel mesh. ITO is 90:10 mix ratio, by weight, of indium-oxide $\left(\mathrm{In}_{2} \mathrm{O}_{3}\right)$ and tin-oxide $\left(\mathrm{SnO}_{2}\right)$, respectively. ITO is widely used in consumer electronics (e.g. touch screens, LCD screens) and available in $1.2 \mathrm{~m}$ wide rolls of ITO-coated mylar foils. The thickness of the ITO coating determines electrical and optical properties of the foil: we use a surface resistance of $15 \mathrm{Ohm} / \mathrm{cm}^{2}$, which gives light transmittance of around $80 \%$. In the third design shown in Figure 1 ), we use two meshes to create the amplification field and the same fluorocarbon wires to separate the planes. The transparency of electrodes can potentially increase the gain by almost a factor of two due to simultaneous readout of two drift volumes.

The experimental setup with a time-projection chamber (TPC) and a CCD camera for optical readout [15] of the amplification plane is shown in Figure 2. The cathode mesh used to create the drift field has a periodicity of $312 \mu \mathrm{m}$ and wire thickness of $31 \mu \mathrm{m}$, which gives an optical transparency of $88 \%$. The drift distance is limited to approximatelly $\pm 5 \mathrm{~cm}$ by the size of the vacuum 
vessel. The CCD camera is manufactured by Finger Lake Instrumentation and equipped with a Kodak KAF-0401ME chip with a cooler that maintains the temperature in the range $[-20,-18] \mathrm{C}$ to minimize electronic noise (25 ADU). The photographic lens has an aperture ratio, f/\# of 0.95 , and a focal length of $25 \mathrm{~mm}$.

a) mesh-copper

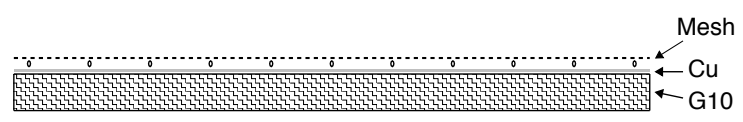

b) mesh-ITO film

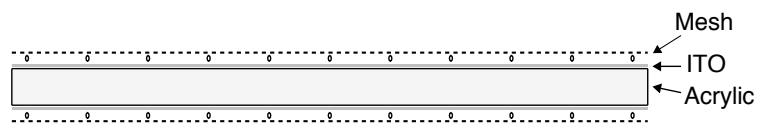

c) mesh-mesh

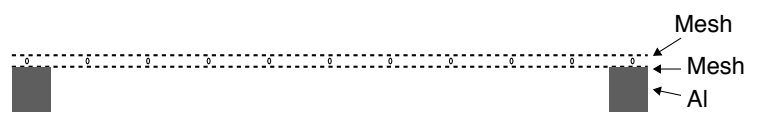

Fig. 1. Schematics of amplification regions formed with a) mesh and a copper sheet, b) mesh and ITO-film, and c) two meshes. In all cases the electrodes are separated with $0.54 \mathrm{~mm}$ fluorocarbon wires.

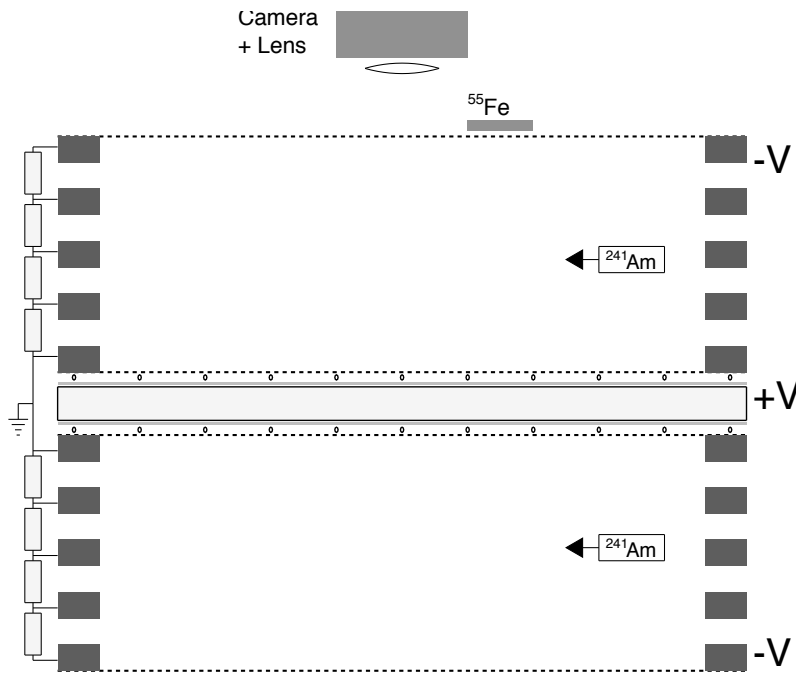

Fig. 2. A schematic of the TPC chamber used in these studies. The inner diameter of the chamber is $23 \mathrm{~cm}$, and the height of a drift region is $5 \mathrm{~cm}$. The ITO-mesh configuration has two drift regions that can be read out simultaneously. 


\section{Results}

\subsection{Detector gain}

We measure the detector gain using $5.5 \mathrm{MeV} \alpha$ particles from a collimated ${ }^{241} \mathrm{Am}$ source. The chamber is filled with $\mathrm{CF}_{4}$ at various pressures in the range 50-200 Torr. The drift field is $500 \mathrm{~V} / \mathrm{cm}$, and the amplification voltage ranges between 0.65 and $1.1 \mathrm{kV}$. Images are taken sequentially with $500 \mathrm{~ms}$ exposure time and resolution of $96 \times 64$ pixels, where each pixel is $488 \times 488 \mu \mathrm{m}^{2}$ $\left(72 \times 72 \mu \mathrm{m}^{2}\right.$ on chip). Pixels that have intensity greater than 5 standard deviations from the mean dark field at least $10 \%$ of the time are flagged as 'hot channels' and excluded in the data analysis. ADC bias is corrected for by subtracting from each image the average of 100 images taken with the shutter closed.

The gain of the detector is determined from the intensity of scintillation light recorded by the CCD camera in a $5 \mathrm{~mm}$ track segment close to the alpha source. The stopping power of alpha tracks in this region is approximately uniform and we estimate it using the SRIM [17] program, as $0.75 \mathrm{MeV} /\left(\mathrm{mg} / \mathrm{cm}^{2}\right)$, which corresponds to 87, 130, 175, 264 and $355 \mathrm{keV}$ of energy loss at 50, 75, 100, 150 and 200 Torr of $\mathrm{CF}_{4}$ pressure, respectively.

We convert the observed light intensity into charge gain by accounting for the transmittance of the amplification and drift meshes (70\%), vessel window (90\%), camera lens and window (90\%). Scintillation light created in the lower drift region has an additional transmittance loss of approximately $60 \%$ as it passes through ITO layers and acrylic plate, or two amplification meshes. We compute the lens acceptance to be $1.1 \cdot 10^{-3}$ and estimate the average CCD efficiency as $40 \pm 10 \%$ using the manufacturer's quantum efficiency curve and the scintillation spectrum of $\mathrm{CF}_{4}$ gas [12]. The gain of the camera is measured to be $1.6 \mathrm{ADU} / \mathrm{e}^{-}$. The ratio of the total number of scintillation photons to the electrons in the avalanche is taken as $1 / 3$ [11]12. We use $w=54 \mathrm{eV}$ for the average ionization energy in $\mathrm{CF}_{4}$ gas, or 18.5 ionization $\mathrm{e}^{-}$per $\mathrm{keV}$ of energy loss [16. Hence, the total gain, $g$ is computed from observed number of counts per keV of energy loss, $I_{C C D}$ as $g=I_{C C D} / 2.5 \cdot 10^{-3}$.

We measure the gain in the mesh-copper detector (Figure 11a) and plot the observed intensity $I_{C C D}$ as a function of the amplification voltage and pressure in Figure 3a). The voltage is increased until the total charge created in the amplification region reaches a sparking threshold. The maximum gain varies from 15-45 ADU/keV, which is roughly 13 times larger than charge amplification achieved with wires [9], after accounting for different light collection efficiencies. Since the lower ionization density allows larger charge multiplica- 
tion, the maximum gain increases with decreasing pressure, as is evident in Figure 3 a). The maximum electronic stopping power of alpha particles occurs in the Bragg peak at around $800 \mathrm{keV}$. The same electronic stopping power is reached at $250 \mathrm{keV}$ for fluorine ions, presenting an upper-energy threshold for detection of nuclear recoils at a given gain. We also compare different gaps between anode and ground planes using different widths of fishing lines. After adjusting the amplification voltage, we find that the separation has no effect on the maximum achievable gain, which is limited by the total charge created in the amplification region.

The gain (or reduction in the number of CCD cameras) can be further improved by placing drift regions opposite to each other along with transparent amplification electrodes, as shown in designs with ITO and all-mesh electrodes in Figures $1 \mathrm{~b}, \mathrm{c}$ ). Two ${ }^{241} \mathrm{Am}$ sources are placed in opposite drift regions approximately $1 \mathrm{~cm}$ from the amplification region, which is made of an ITO-mesh sandwich (Figure 1b). Both drift regions are read out by the same CCD camera as shown in Figure 2. An image taken with a 500 ms-exposure of several alpha tracks from two ${ }^{241} \mathrm{Am}$ sources is shown in Figure 4 . Note that the signal from tracks in the lower source is attenuated due to passage through two ITO-coated anodes and a ground mesh. We measure the attenuation to be $(52 \pm 2) \%$, which is close to the expected attenuation of $(58 \pm 1) \%$ when we assume the same gain for the upper and lower amplification regions. We evaluate the gain using the signal from the upper source, and plot it in Figure $3 \mathrm{~b}$ ). The gain is found to be 20-30\% lower than with the copper-mesh amplification plane. Further improvements are possible with foils that have higher resistivity (light transmittance) and better quality control during shipment and module production, as our current foils arrived slightly damaged.

Finally, we test a detector that has the amplification plane made entirely of meshes. The gain measurement is shown in Figure 3c). Values are comparable with ITO-based detector and approximately 30\% lower than the copper-mesh detector. Operation with a readout of two drift regions can be realized by adding a third mesh.

An energy resolution of approximately $10 \%$ and spatial resolution of $400 \mu \mathrm{m}$ are measured here and found to be comparable with our previous measurements using MWPC [9]. Contribution to the spatial resolution from the diffusion of electrons that are $2 \mathrm{~cm}$ above the amplification plane is $140 \mu \mathrm{m}$, finite CCD bin size adds $488 / \sqrt{12} \approx 140 \mu \mathrm{m}$, and finite mesh pitch adds $256 / \sqrt{12} \approx 74 \mu \mathrm{m}$ 
a) mesh-copper

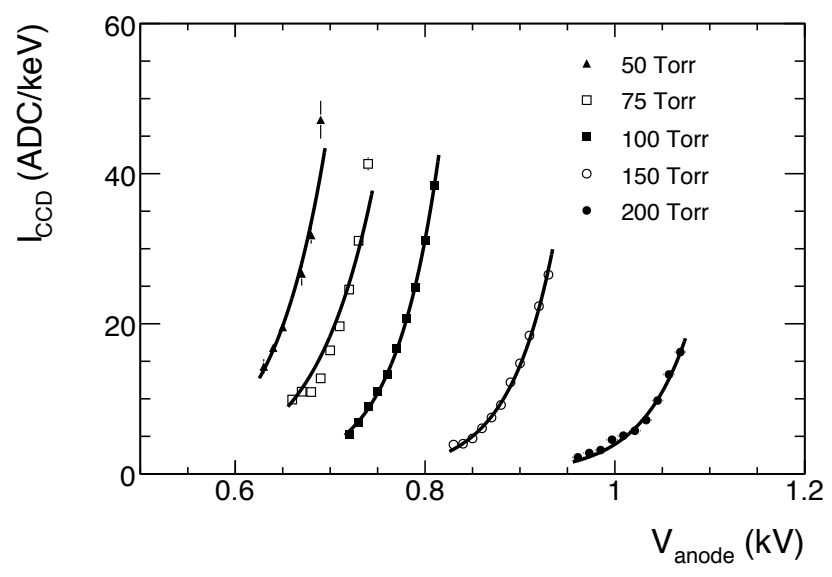

b) mesh-ITO film

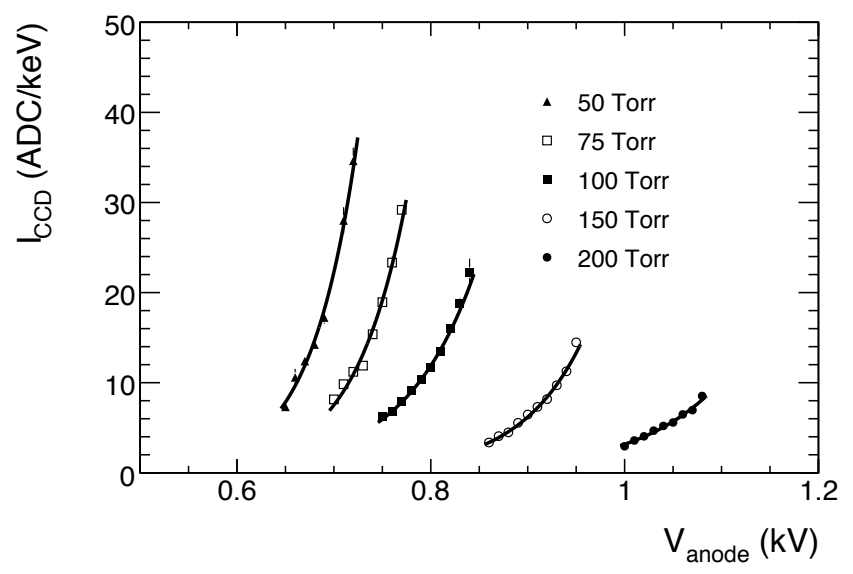

c) mesh-mesh

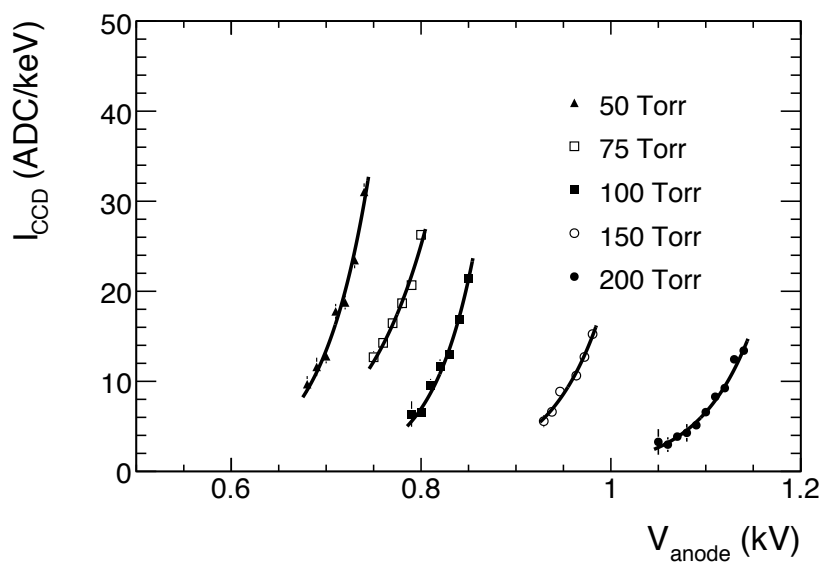

Fig. 3. The gain measured in the three amplification designs. 


\subsection{Gain uniformity}

The uniformity of the gain is demonstrated by observing a clear Bragg peak in mesh-ITO and mesh-copper detector designs (Figure 1 $1 \mathrm{a}, \mathrm{b}$ ). A scintillation profile of an alpha track that has a range of approximately $4.5 \mathrm{~cm}$ in 250 Torr of $\mathrm{CF}_{4}$ is shown in Figure 4 .

In the case of the mesh-mesh detector (Figure 1,), gain non-uniformity can occur on a smaller scale if the relative alignment between the meshes varies across the surface of the detector. The charge multiplication and light collection are more efficient if wires of the anode mesh fall in between wires of the ground mesh, i.e. with offset of half of a mesh pitch in both $x$ and $y$ directions. The variation in relative alignment between the two meshes may be the result of a non-zero relative angle, non-uniform mesh tensioning, or variation in spacing between the mesh planes. We show an example of this by taking a photograph of light scattered from the bottom mesh and transmitted through the upper mesh, as shown in Figure 5a). A moiré pattern with periodicity of roughly $2 \mathrm{~mm}$ is created by the change in relative alignment between two meshes. In order to evaluate the effect on the gain, we expose the detector to $\mathrm{x}$-rays from an ${ }^{55} \mathrm{Fe}$ with gas pressure set to 400 Torr and anode voltage to $1.74 \mathrm{kV}$. The average gain of approximately $9 \mathrm{ADU} / \mathrm{keV}$ is determined from the total light emitted by $5.5 \mathrm{MeV}$ alpha particles. An ${ }^{55} \mathrm{Fe}$ source is mounted on the top of the cathode mesh and several minutes of 1-second exposures are taken, with accumulated CCD image shown in Figure 5b). The average light intensity decreases with distance from the source due to the reduced flux of x-rays. The semi-circular shadow on the right is due to the Fe source,

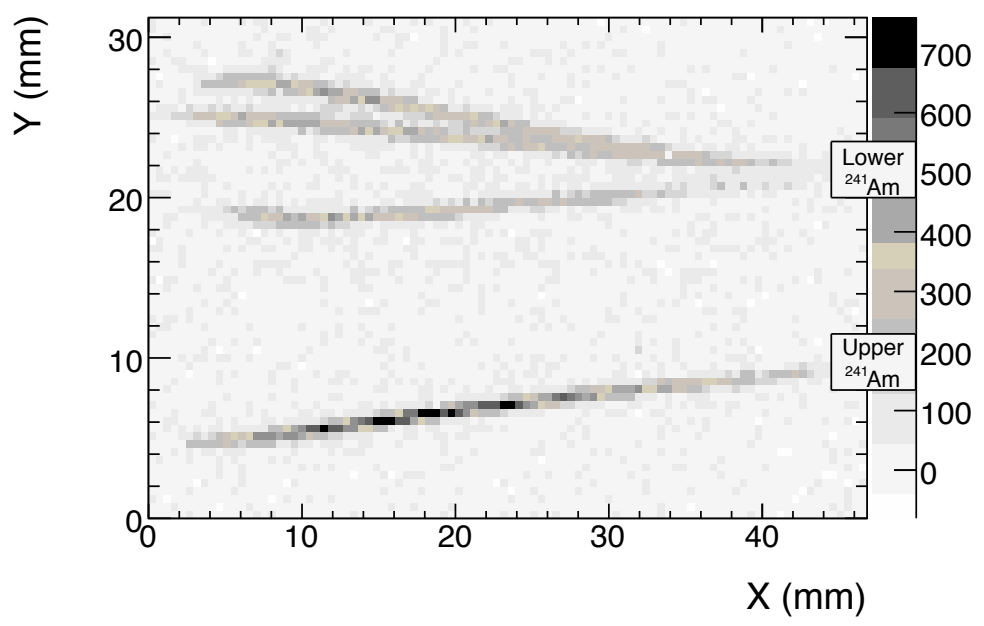

Fig. 4. An image of alpha tracks from $500 \mathrm{~ms}$ exposure at 200 Torr with two ${ }^{241} \mathrm{Am}$ sources placed in opposite drift regions. The amplification electrodes are mesh and ITO foil. 
and the long shadows are due to fishing line separators. The checkered light pattern seen in the plot is due to variation of the gain and follows the moiré pattern. Using pixels that have equal distance from the source, we estimate a gain variation to be approximately $50 \%$ between maxima and minima. The non-uniformity in gain can be minimized, if not avoided, by applying extra care during fabrication of detector. Elimination of the moiré pattern during the mesh stretching and assembly can be used as a monitor for quality control. Any remaining gain variation can be accounted for by calibrating with x-ray sources.

a) Moiré pattern

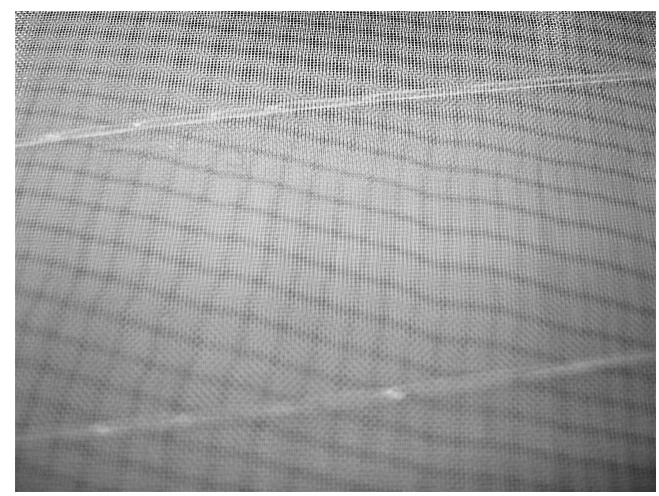

b) ${ }^{55} \mathrm{Fe}$ exposure

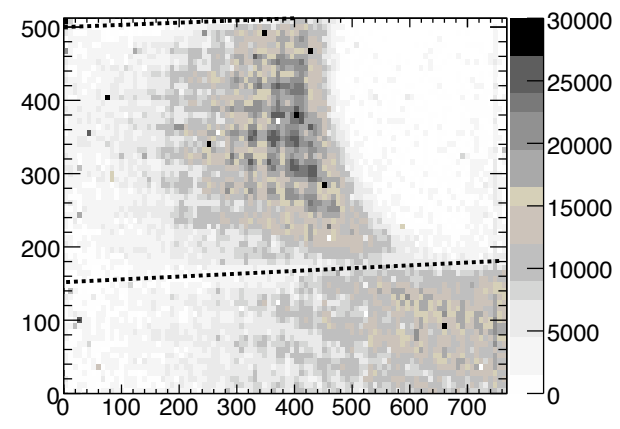

Fig. 5. Evaluation of gain uniformity in the mesh-mesh detector using moiré pattern and ${ }^{55} \mathrm{Fe}$ exposure. The distance between horizontal resistive wires is $2 \mathrm{~cm}$.

\section{3 "Head-tail" effect}

Finally, we demonstrate the ability to determine the directional sense of lowmomentum nuclei, the "head-tail" effect. The ionization rate by a low-momentum nucleus decreases as it slows down in the detector gas, and the sense of its direction can be deduced from the scintillation profile along the track. We place a ${ }^{252} \mathrm{Cf}$ source approximately $2 \mathrm{~m}$ from the detector's view-field of $4.8 \times 3.2 \mathrm{~cm}^{2}$, resulting in a well determined direction of the neutron flux. We use the coppermesh amplification plane (see Figure 1 1 ) with the anode voltage set to $740 \mathrm{~V}$ and the $\mathrm{CF}_{4}$ pressure at 75 Torr. Typical ranges for nuclear recoils created in neutron scattering are of the order of a few millimeters.

Taking 6000 1-second exposures without a trigger, we search for clusters of pixels that are 3 standard deviations above the CCD noise. In most cases there is only one cluster per event, but otherwise we select the most energetic cluster. The energy is computed from the sum of pixel yields after background subtraction, using calibration resulting from inserting an alpha source three times: before, in the middle and after the neutron exposure. We find an average gain of $27 \mathrm{ADU} / \mathrm{keV}$. The gain decreases by $8 \%$ during the measurements, probably 
due to air leakage into the vacuum vessel. We describe quenching correction for a recoil with energy $E$ in terms of electronic $S_{e}(E)$ and nuclear $S_{n}(E)$ stopping powers, $q(E) \approx\left(S_{e}(E)+S_{n}(E) \cdot 0.3\right) /\left(S_{e}(E)+S_{n}(E)\right)$, where factor 0.3 is chosen to be small and non-zero. The quenching correction, $q$, which is about $25 \%$ at $100 \mathrm{keV}$ and $3 \%$ at $900 \mathrm{keV}$ [17,18], is used in the calculation of light output for fluorine recoils. The range of the recoil track is determined as the maximum distance between the pixels in the cluster. Recoils are defined to have at least $6 \mathrm{CCD}$ bins that are not touching the boundary of the CCD view field. We require a ratio of principal moments of inertia greater than 5, which imposes a minimum energy cut of around $100 \mathrm{keV}$ on nuclear recoils. Discharge events are removed with a cut on the maximum light per event, and remaining spot-like events are removed with the cut on the ration of principal moments. A plot of energy versus range of simulated events and data is shown in Figure 6 for recoil candidates that have reconstructed energy above $100 \mathrm{keV}$. The simulation is based on cross sections found in a nuclear scattering library [19] and fluorine ion propagation determined from the SRIM [17].
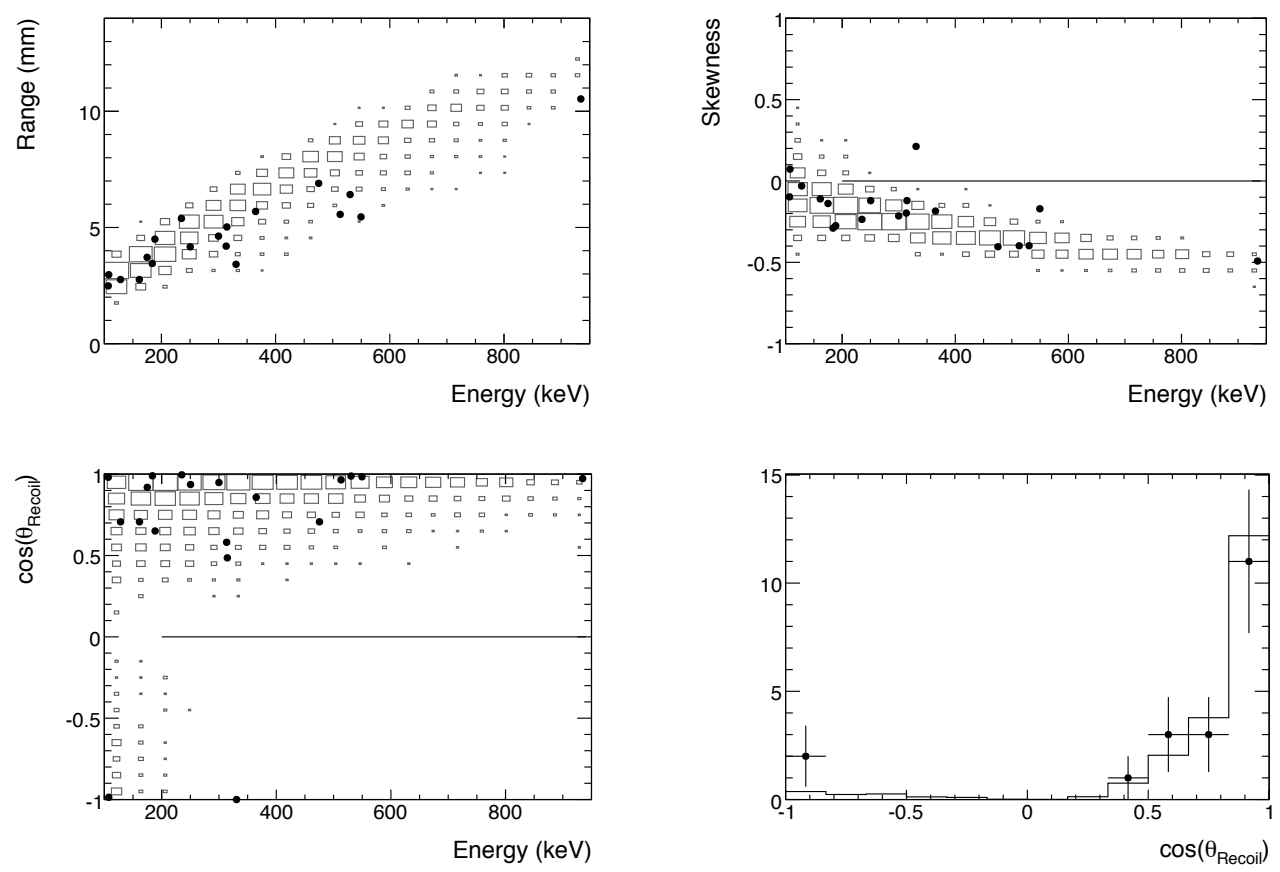

Fig. 6. Range (top left), skewness (top right), and cosine of 2D recoil angle (bottom left) vs. reconstructed energy for nuclear recoil candidates in a ${ }^{252} \mathrm{Cf}$ exposure at 75 Torr. Black points are data, the box-histogram is simulation. Signed distribution of the cosine of $2 \mathrm{D}$ recoil angle (bottom right), with data and simulation normalized to the same area.

We show images of recoil tracks with highest energies in Figure 7) In all images neutrons are incident from the right, along the $x$ axis. The nuclear recoils also propagate from the right, and we observe a decreasing light intensity as the recoils slow down in the $\mathrm{CF}_{4}$ gas, as expected. We quantify the scintillation 
asymmetry along the track using skewness, which is described previously in more detail [9]. It is a dimensionless ratio of the third moment and the rootmean-square cubed of the light distribution along the track. Negative skewness is expected for recoils heading in the direction of the neutron beam. The topright plot in Figure 6 shows the skewness distribution for tracks in data and simulation.

The two pixels with maximum separation that are used in the range measurement are also used in the calculation of the recoil angle. Figure 8 shows the resolution for the recoil angle based on the simulation. The sense of direction

is determined from the "head-tail" (skewness) measurement. We show for the first time, distributions for the signed cosine of the $2 \mathrm{D}$ recoil angle, $\cos \theta_{\text {Recoil }}$ vs. energy and signed cosine of the 2D recoil in Figure 6.

We define a quality factor, $Q_{H T}$ for the "head-tail" asymmetry at given recoil energy, $E_{R}$ as

$$
Q_{H T}\left(E_{R}\right)=\varepsilon\left(E_{R}\right) \cdot\left(1-2 \omega\left(E_{R}\right)\right)^{2}
$$

where the recoil reconstruction efficiency, $\varepsilon$ and the fraction of wrong "headtail" assignments, $\omega$ are determined from simulation. The $Q_{H T}$ is the effective fraction of reconstructed recoils with "head-tail" information, and the error on the "head-tail" asymmetry scales as $1 / \sqrt{Q_{H T}}$. Figure 8 shows the quality factor in the energy range that has been explored so far.

\section{Conclusion and outlook}

Directional detection of dark matter requires large detector volumes with fine granularities. TPC modules filled with low-pressure $\mathrm{CF}_{4}$ gas can provide directionality and "head-tail" directional sense, in particular for spin-dependent dark matter searches. We have demonstrated three possibilities for chargeamplification that allow directional detection and "head-tail" determination. In all three cases the gain is improved by more than an order of magnitude compared to the previous MWPC design [9]. This results in improvement in the "head-tail" discrimination due to the larger gain, 2D detection of recoil images, and reduced pressure from 200 to 75 Torr, allowing for longer recoil tracks. We estimate the sensitivity for the WIMP detection using standard assumptions about the dark matter halo [21]. Cross-section limits for spin-dependent WIMP scattering on proton are shown in Figure 9. We can improve current experimental limits [4] with approximately $0.1 \mathrm{~kg} \cdot \mathrm{y}$ of $\mathrm{CF}_{4}$ exposure, and test MSSM models [22] with approximately $100 \mathrm{~kg} \cdot \mathrm{y}$. In both cases we assumed operation in an underground laboratory with a neutroninduced background rate of 0.01 events $/(\mathrm{keV} \cdot \mathrm{kg} \cdot \mathrm{y})$ [20], uncorrelated with 
Cygnus direction, and a $50 \mathrm{keV}$ recoil-energy threshold. We plan to pursue these technologies and construct a cubic-meter module that will be a basic building block of a ton-scale detector.

\section{Acknowledgments}

We wish to thank the Office of Environment, Health \& Safety at MIT for supplying radioactive sources, the Laboratory for Nuclear Science MIT for technical support. We thank Akira Hitachi and Jeff Filippini for helpful discussions, and Gus Zhang of Prochema for providing samples of ITO-mylar

a) $935 \mathrm{keV}$

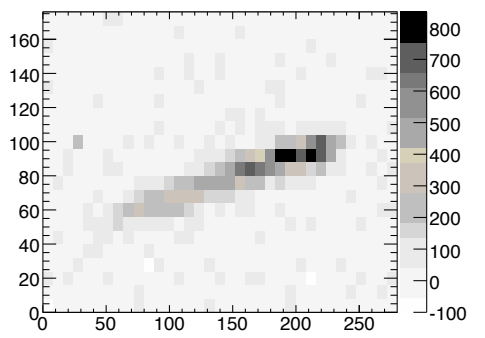

a) $530 \mathrm{keV}$

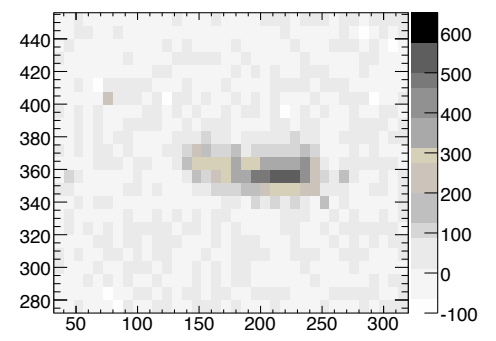

c) $365 \mathrm{keV}$

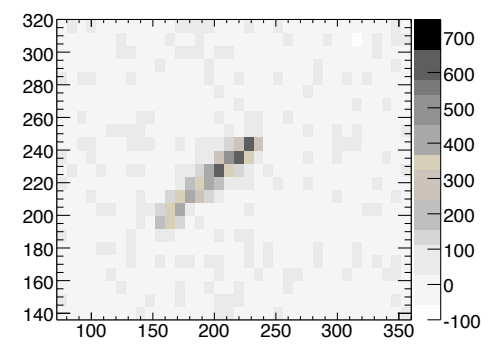

b) $550 \mathrm{keV}$

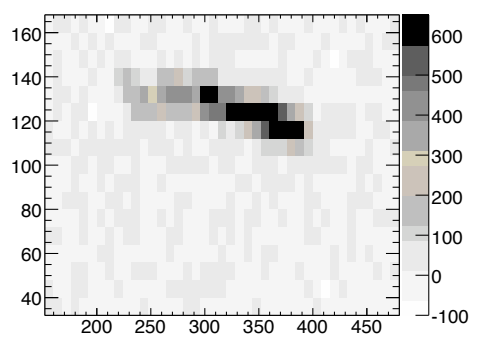

b) $475 \mathrm{keV}$

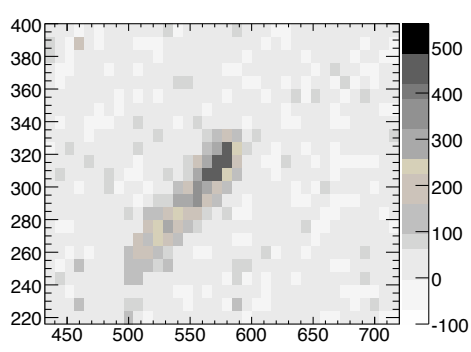

d) $313 \mathrm{keV}$

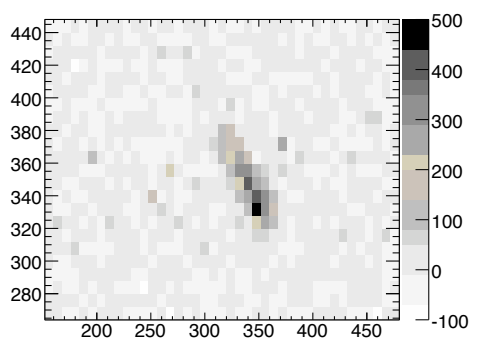

Fig. 7. Scintillation profile of nuclear recoil candidates in a ${ }^{252} \mathrm{Cf}$ exposure at 75 Torr. Neutrons are incident from the right, along the $x$ axis. 
foils. We acknowledge support by the Advanced Detector Research Program of the U.S. Department of Energy (contract number 6916448), the Reed Award Program, the Ferry Fund, the Pappalardo Fellowship program, the MIT Kavli Institute for Astrophysics and Space Research, and the Physics Department at the Massachusetts Institute of Technology.

\section{References}

[1] R. J. Gaitskell, Ann. Rev. Nucl. Part. Sci. 54, 315 (2004).

[2] R. Bernabei et al. [DAMA Collaboration], arXiv:0804.2741 [astro-ph].

[3] Recent limits on spin-independent WIMP interactions: J. Angle et al. [XENON Collaboration], Phys. Rev. Lett. 100, 021303 (2008); Z. Ahmed et al. [CDMS Collaboration], arXiv:0802.3530 [astro-ph].

[4] Recent limits on spin-dependent WIMP interactions: G. J. Alner et al. [UK Dark Matter Collaboration], Phys. Lett. B 616, 17 (2005); E. Behnke et al. [COUPP
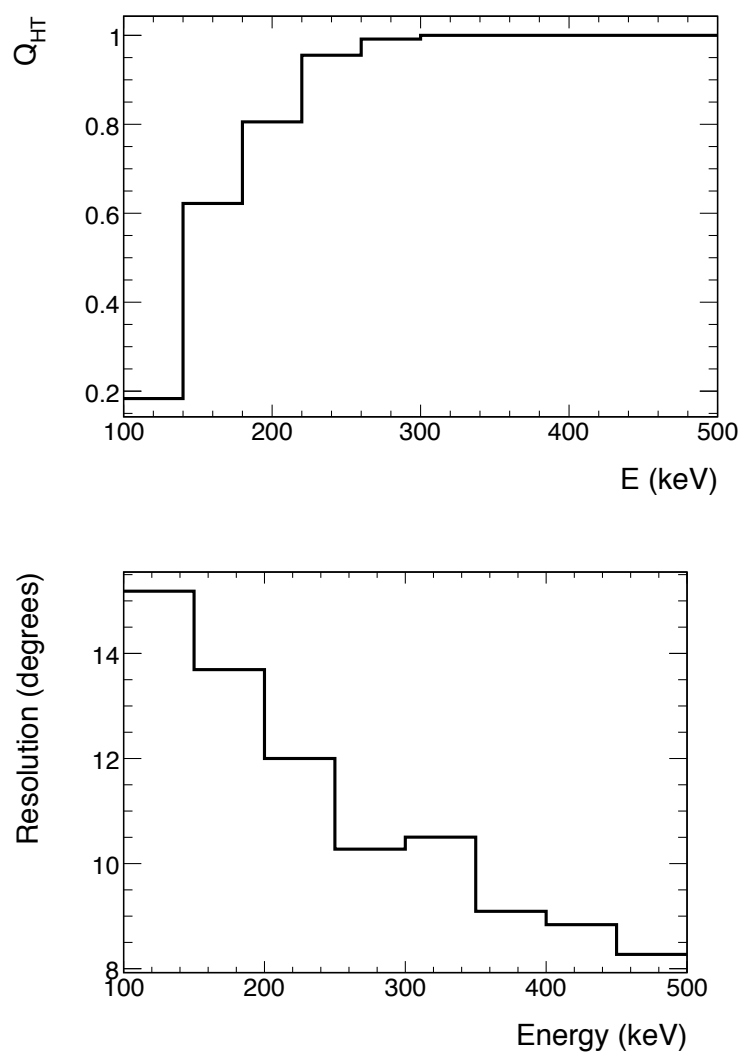

Fig. 8. Quality factor for the "head-tail" measurement vs. reconstructed energy (top), and 2D angular resolution (bottom) based on simulation for neutron scattering experiment. 
Collaboration], Science 319, 933 (2008); H. S. Lee. et al. [KIMS Collaboration], Phys. Rev. Lett. 99, 091301 (2007).

[5] D. N. Spergel, Phys. Rev. D 37, 1353 (1988); A. M. Green and B. Morgan, Astropart. Phys. 27, 142 (2007); O. Host and S. H. Hansen, JCAP 0706, 016 (2007); M. S. Alenazi and P. Gondolo, Phys. Rev. D 77, 043532 (2008).

[6] S. Burgos et al., Astropart. Phys. 28, 409 (2007). P. K. Lightfoot, N. J. C. Spooner, T. B. Lawson, S. Aune and I. Giomataris, Astropart. Phys. 27, 490 (2007); D. P. Snowden-Ifft, C. J. Martoff and J. M. Burwell, Phys. Rev. D 61, 101301 (2000); C. J. Martoff, D. P. Snowden-Ifft, T. Ohnuki, N. Spooner and M. Lehner, Nucl. Instrum. Meth. A 440, 355 (2000).

[7] K. Miuchi et al., Phys. Lett. B 654, 58 (2007); T. Tanimori, H. Kubo, K. Miuchi, T. Nagayoshi, R. Orito, A. Takada and A. Takeda, Phys. Lett. B 578, 241 (2004).

[8] D. Santos, O. Guillaudin, T. Lamy, F. Mayet and E. Moulin, J. Phys. Conf. Ser. 65, 012012 (2007).

[9] D. Dujmic et al., Nucl. Instrum. Meth. A 584, 327 (2008).

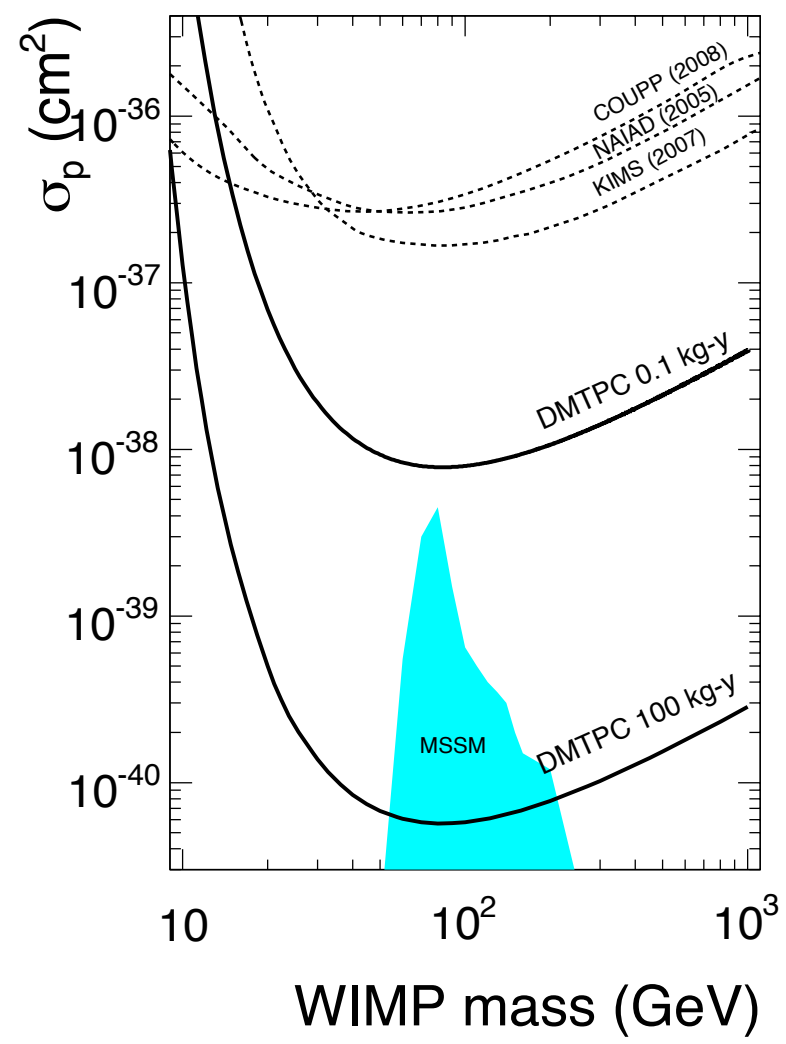

Fig. 9. Estimated sensitivity (90\% C.L.) for spin-dependent WIMP scattering with a mesh detector. We expect that MSSM models can be tested with approximately $100 \mathrm{~kg} \cdot \mathrm{y}$ of target mass-exposure in an underground laboratory. 
[10] P. G. Datskos, J. G. Carter, and L. G. Christophorou, J. Appl. Phys. 71 (1982) 15.

[11] A. Pansky, A. Breskin, A. Buzulutskov, R. Chechik, V. Elkind and J. Va'vra, Nucl. Instrum. Meth. A 354, 262 (1995).

[12] A. Kaboth et al., arXiv:0803.2195 [physics.ins-det].

[13] G. Charpak, R. Bouclier, T. Bressani, J. Favier and C. Zupancic, Nucl. Instrum. Meth. 62, 262 (1968).

[14] F. Angelini, R. Bellazzini, A. Brez, M. M. Massai, R. Raffo, G. Spandre and M. A. Spezziga, Nucl. Instrum. Meth. A 335, 69 (1993); Y. Giomataris, P. Rebourgeard, J. P. Robert and G. Charpak, Nucl. Instrum. Meth. A 376, 29 (1996); F. Sauli, Nucl. Instrum. Meth. A 386, 531 (1997).

[15] F.A.F. Fraga et al.. Nucl. Instr. and Meth. A 471 (2001), p. 125

[16] A. Sharma, SLAC-JOURNAL-ICFA 16 (1998) 3.

[17] J. F. Ziegler, J. P. Biersack, U. Littmark, Pergamon Press, New York, 1985. The code is available online at www.SRIM.org.

[18] A. Hitachi, arXiv:0804.1191.

[19] M.B. Chadwick et al., Nuclear Data Sheets 107 (2006), 2931-3060

[20] D. Mei and A. Hime, Phys. Rev. D 73, 053004 (2006).

[21] J. D. Lewin and P. F. Smith, Astropart. Phys. 6, 87 (1996).

[22] J. R. Ellis, A. Ferstl and K. A. Olive, Phys. Rev. D 63, 065016 (2001). 